\title{
PT SYMMETRY IN QUANTUM PHYSICS: FROM A MATHEMATICAL CURIOSITY TO OPTICAL EXPERIMENTS
}

. Carl M. Bender - Washington University in St. Louis, St. Louis, MO 63130, USA - DOI: http://dx.doi.org/10.1051/epn/2016201

Space-time reflection symmetry, or PT symmetry, first proposed in quantum mechanics by Bender and Boettcher in 1998 [1], has become an active research area in fundamental physics. More than two thousand papers have been published on the subject and papers have appeared in two dozen categories of the arXiv. Over two dozen international conferences and symposia specifically devoted to $\mathrm{PT}$ symmetry have been held and many PhD theses have been written.

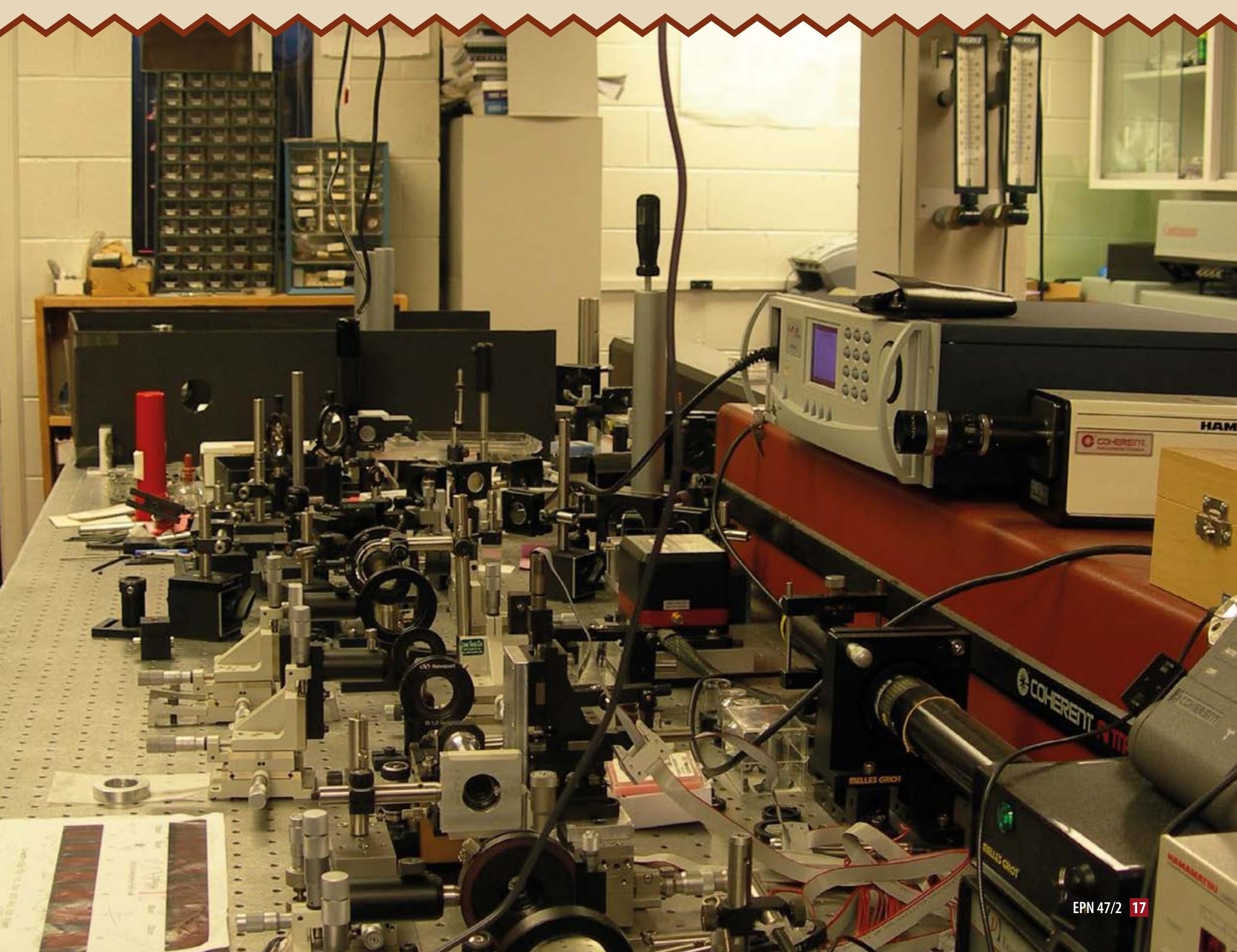


4. 17: The first tabletop optics experiment on PT symmetry. This experiment is described in Ref. [5] (Photo taken by Carl Bender during a visit to the laboratory.)

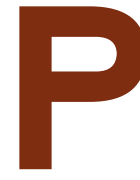

T-symmetric quantum mechanics is an extension of conventional quantum mechanics into the complex domain. (PT symmetry is not in conflict with conventional quantum theory but is merely a complex generalization of it.) $\boldsymbol{P T}$-symmetric quantum mechanics was originally considered to be an interesting mathematical discovery but with little or no hope of practical application, but beginning in 2007 it became a hot area of experimental physics. It has now been explored experimentally in such diverse fields as optical wave guides, lasers, optical resonators, microwave cavities, superconducting wires, NMR, graphene, and metamaterials, and this work has been published in such high-impact journals as Nature, Science, and Physical Review Letters. Using techniques developed in these studies it appears likely that it will be possible to use $\boldsymbol{P T}$ symmetry to develop new ways to control light, perhaps even leading to new kinds of computers that use optical beams instead of electric wires. It may well be used to formulate new kinds of materials and to develop new kinds of communication devices.

\section{Two profound discoveries}

To understand the background of $\boldsymbol{P} \boldsymbol{T}$-symmetric quantum mechanics, recall that two profound discoveries in the early $20^{\text {th }}$ century transformed classical physics into what we regard today as modern physics. The first was quantum mechanics, which describes the nature of matter, the stability of atomic energy levels, the binding of atoms to form molecules, and the properties of materials. The second was relativity, which describes the geometry of space and time. Symmetries are a central component of physical laws, and these theories both possess fundamental symmetries, a discrete symmetry in quantum mechanics called Hermiticity and a continuous symmetry in special relativity, which is expressed in terms of the Poincaré group.

Phenomena such as quantum interference imply that complex numbers play an essential role in explaining physical observations in quantum mechanics. The Schrödinger equation, which is the fundamental equation of quantum mechanics, is complex. It is partly because of complex numbers that quantum theory makes probabilistic rather than definite predictions. (For example, the mass of an unstable particle appears as a pole at a specific point in the complex plane, but on the real axis the remnant of this pole is a probability distribution, and we cannot say exactly what the mass of the particle is.)

\section{Symmetries}

While complex numbers are central in quantum mechanics, the symmetries of space-time are restricted to the real domain. A point in space-time is represented by the real four-dimensional vector $(x, y, z, t)$. The Poincaré group, the continuous symmetry group of space-time, is ten-dimensional: First, there are four translations; repeating an experiment in a laboratory located at different points in space and time $(x+a, y+b, z+c, t+d)$ will yield the same experimental result. Second, there are three rotations; we can rotate about the $x, y$, and $z$ axes. Repeating an experiment in a laboratory that has been rotated in space will yield the same result. Third, we can boost the velocity in three possible ways, along the $x, y$, or $z$ axes. Again, repeating an experiment in a laboratory that is moving at a constant velocity relative to the original laboratory will yield the same result. The homogeneous Lorentz group is defined as the six-parameter group of all real $4 \times 4$ matrices that perform rotations and boosts (not translations) on the space-time vector $(x, y, z, t)$ but leave the numerical value of the Lorentz scalar $x^{2}-t^{2}$ invariant. For many years it was thought that this six-parameter group (plus translations) was the fundamental geometrical symmetry group of the universe. However, along with the continuous transformations (rotations and boosts), the definition of this group allows for two discrete transformations that we now know are not symmetries of nature. The first, called parity $\boldsymbol{P}$, changes the sign of the spatial part of the four vector $P:(x, y, z, t) \rightarrow(-x,-y,-z, t)$. (This symmetry operation changes one's right hand into one's left hand; such a transformation cannot be achieved by a rotation.) The second, called time reversal $T$, changes the sign of the time component of the four vector $T:(x, y, z, t)$ $\rightarrow(x, y, z,-t)$. A Nobel prize was awarded to Lee and Yang in 1957 for demonstrating that parity is not a symmetry of nature and another was awarded to Cronin and Fitch in 1980 for demonstrating that time reversal is also not a symmetry of nature. (A left-handed laboratory can obtain different experimental results from a right-handed laboratory and a laboratory traveling backward in time can obtain different results from a laboratory traveling forward in time.) After these advances, it was accepted that the correct geometrical symmetry of nature must exclude $\boldsymbol{P}$ and $\boldsymbol{T}$.

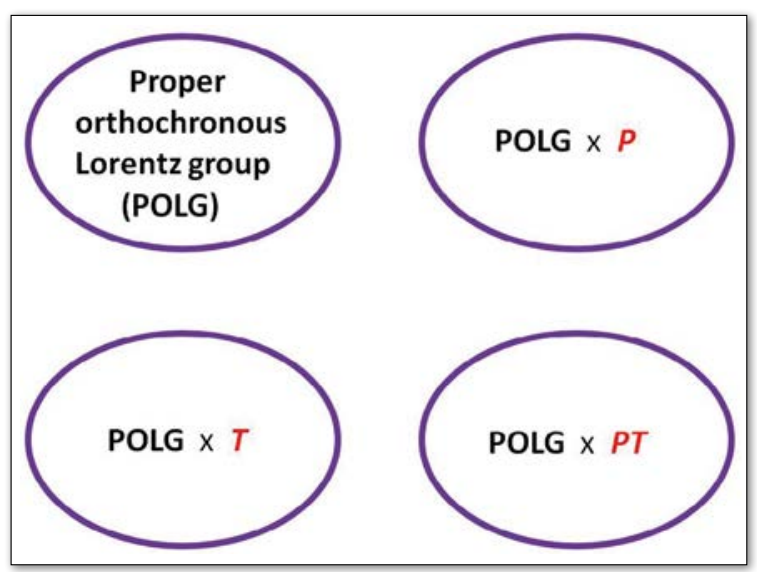

- FIG. 1: Structure of the homogeneous Lorentz group, the group of all real $4 \times 4$ matrices that leave the Lorentz scalar $x^{2}-t^{2}$ invariant. This group consists of four disconnected parts, a subgroup called the proper orthochronous Lorentz group (POLG), and the elements of the POLG multiplied by parity $P$, time-reversal $T$, and space-time reflection $P T$. If the $4 \times 4$ matrices in the Lorentz group are allowed to be complex, we obtain the complex Lorentz group, which has only two disconnected parts. In the complex Lorentz group the POLG is joined continuously to the POLG $\times P T$ and the POLG $\times P$ is joined continuously to the $P O L G \times T$. 


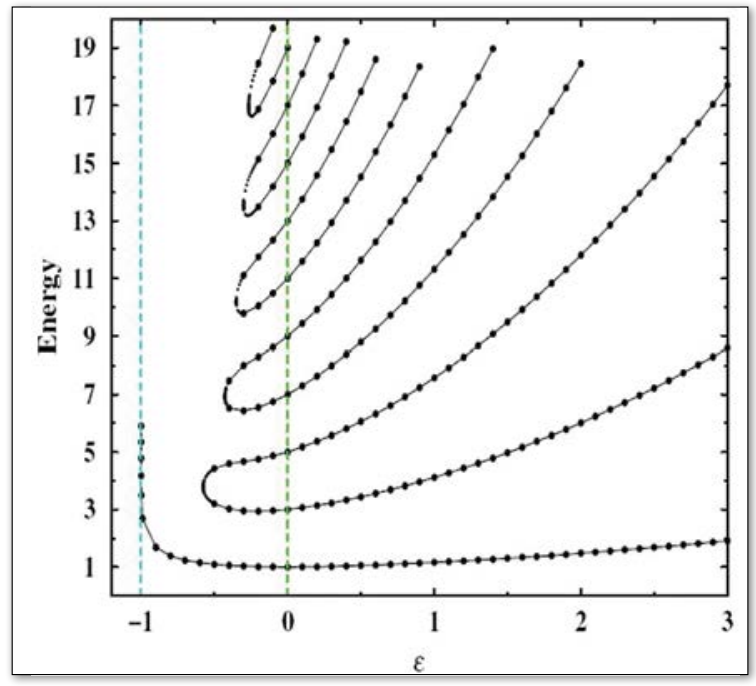

Removing these symmetries from the Lorentz group, we obtain a new smaller symmetry group called the proper orthochronous Lorentz group (see Fig. 1).

\section{Extended Lorentz group}

What happens if we assume that, as in quantum mechanics, complex numbers play a role in geometry? That is, what happens if we extend the proper orthochronous Lorentz group to include complex as well as real $4 \times 4$ matrices? If we extend the Lorentz group to the complex domain, a new discrete symmetry, namely, $P$ T symmetry, emerges naturally. $\boldsymbol{P} \boldsymbol{T}$ symmetry means combined $\boldsymbol{P}$ and $\boldsymbol{T}$ symmetry; a $\boldsymbol{P} \boldsymbol{T}$ reflection changes the sign of all four components of a space-time vector PT: $(x, y, z, t) \rightarrow$ $(-x,-y,-z,-t)$. For uncharged particles that are their own antiparticles this discrete symmetry is a fundamentally correct symmetry of nature. (For fermions and charged particles, $\boldsymbol{P} \boldsymbol{T}$ symmetry is augmented with an additional symmetry operator called charge conjugation $C$, which turns particles into antiparticles. This is the origin of the famous $\boldsymbol{C P T}$ theorem in particle physics.)

Wigner showed that in quantum mechanics the time-reversal operator $\boldsymbol{T}$ changes the sign of the imaginary number $i$. This is because the position operator $x$ and the momentum operator $p$ obey the Heisenberg algebra: $x p-p x=i$. (This fundamental equation leads to the famous Heisenberg uncertainty principle.) Since the momentum $p$ changes sign (particles reverse direction) under time reversal while the position $x$ does not, $i$ changes sign in order to preserve the Heisenberg algebra. Thus, a $\boldsymbol{P T}$-invariant combination (one that does not change sign under $\boldsymbol{P T}$ ) is $i x$, and any quantum theory that contains the combination ix is $\boldsymbol{P T}$ invariant.

The very first $\boldsymbol{P} \boldsymbol{T}$-symmetric quantum theory, which was proposed in 1998 [1], was defined by the Hamiltonian $H=p^{2}+x^{2}(i x)^{\varepsilon}$ ( $\varepsilon$ is a real parameter) in which the combination $(i x)$ appears. The notable feature of this Hamiltonian is that it is not Hermitian and one might think that such a theory should be immediately rejected on this basis.
4 FIG. 2: Graph that launched PT-symmetric quantum theory. The energy levels of the quantummechanical Hamiltonian $H=p^{2}+x^{2}(i x)^{\varepsilon}$ are plotted versus real parameter $\varepsilon$. This Hamiltonian is a complex deformation of the harmonic-oscillator Hamiltonian. It is astonishing that when $\varepsilon>0$, all the energy levels are real even though the Hamiltonian is non-Hermitian. This is because $H$ is $P T$ symmetric. When $\varepsilon<0$, the energies join in pairs and become complex (not shown). When $\varepsilon=0$, $H$ reduces to the Hamiltonian for the harmonic oscillator, whose energy levels are 1, 3, 5, 7,...

However, it was shown that the theory defined by this Hamiltonian has the two essential properties of a conventional quantum theory: First, the energy levels (eigenvalues of $H$ ) are all real and positive if $\varepsilon$ is positive. (The energies of this Hamiltonian are plotted in Fig. 2.) The reality of the energy levels for $\varepsilon>0$ was proved rigorously in 2001 by Dorey, Dunning, and Tateo [2]. Second, probability is conserved in time. This condition, called unitarity, was established in 2002 by Bender, Brody, and Jones [3].

\section{Upside-down potential}

The graph in Fig. 2 is remarkable. It shows that the Hamiltonian $H=p^{2}+i x^{3}$, obtained by setting $\varepsilon=1$, actually has real positive energy levels even though it is complex. Moreover, the Hamiltonian $H=p^{2}-x^{4}$, obtained by setting $\varepsilon=2$, also has real positive energy levels. This is particularly surprising because $-x^{4}$ is an upside-down potential! One might think that a classical particle located at $x=0$ and subject to such a potential would slide down to infinity (unless it was precariously balanced at the top of the potential hill). This does indeed happen. However, the particle does not remain at infinity! Complex analysis shows that the particle repeatedly slides right back up to the top of the hill, and actually spends most of its time there. It is extremely unlikely to find the classical particle far from the origin; it is most probable to find the classical particle near $x=0$. At the quantum level a particle in this upside-down potential is in a bound state strongly localized at the origin. The explanation for this surprising behavior is that we have extended real space to complex space. Complex numbers differ from real numbers in that the complex numbers are not ordered. If $a$ and $b$ are real numbers, we can say that $a>b$ or $b>a$. However, even though the real numbers are embedded in the complex numbers, we cannot say that one complex number is greater than another complex number, so it makes no sense to say that the "top" of the potential is at $x=0$ ! One must think in new ways when working in the complex domain.

We learn from this model that quantum theories need not obey the conventional mathematical condition of Hermiticity so long as they obey the physical geometric condition of space-time-reflection symmetry (PT symmetry). $\boldsymbol{P} \boldsymbol{T}$ symmetry challenges a standard convention in physics - the widely held belief that a quantum Hamiltonian must be Hermitian. And, because $\boldsymbol{P T}$ symmetry is a weaker condition than Hermiticity, there are infinitely many Hamiltonians that are $\boldsymbol{P} \boldsymbol{T}$ symmetric but non-Hermitian; we can now study new kinds of quantum theories that would have been rejected in the past as being unphysical. Moreover, $\boldsymbol{P T}$-symmetric systems exhibit a 


\begin{tabular}{|cc|}
\hline FIG.3: PT-symmetric optical wave \\
guide. One wave guide has gain; this \\
is accomplished by shining a laser on \\
an optically active material such as \\
$\begin{array}{r}\text { lithium niobate. The other wave guide } \\
\text { has loss, which is accomplished by } \\
\text { plating the wave guide with a metal } \\
\text { such as chromium. The wave guides are } \\
\text { coupled by placing them in close physical } \\
\text { proximity so that they can exchange } \\
\text { energy and achieve equilibrium. }\end{array}$
\end{tabular}

feature that Hermitian systems cannot; as indicated in Fig. 1, the energy levels become complex when $\varepsilon<0$. The transition from real to complex energies is a key feature of $\boldsymbol{P T}$-symmetric systems and it is called the $\boldsymbol{P T}$ phase transition. At this transition the system goes from a state of physical equilibrium (called a state of unbroken PT symmetry) to nonequilibrium (broken $\boldsymbol{P} \boldsymbol{T}$ symmetry).

\section{Balanced loss and gain}

$\boldsymbol{P T}$-symmetric systems typically have complex potentials and thus can be thought of as non-isolated systems interacting with their environment. A potential with a positive-imaginary part describes a system that receives energy from its environment; a potential with a negative-imaginary part describes a system that loses energy to its environment. However, a $\boldsymbol{P T}$-symmetric system is special; the condition of $\boldsymbol{P T}$ symmetry means that loss and gain are exactly balanced. One can fabricate a $\boldsymbol{P T}$-symmetric system in the laboratory by coupling two identical subsystems, one with gain and the other with loss. (The composite system is $\boldsymbol{P T}$ symmetric because space reflection $\boldsymbol{P}$ interchanges the subsystems and time reversal $\boldsymbol{T}$ switches the roles of gain and loss.) Many experiments, mostly in optics, have readily observed the $\boldsymbol{P} \boldsymbol{T}$ phase transition between regions of broken and unbroken $\boldsymbol{P T}$ symmetry.

The connection between $\boldsymbol{P T}$-symmetric quantum mechanics and optics was proposed by El-Ganainy et al. [4]. The connection is simply that the equation describing the paraxial ray (the wave in the center) of a wave guide satisfies an equation of exactly the same form as the Schrödinger equation except that time $t$ is replaced by $z$, the distance along the wave guide, and the potential $V$ is replaced by the refractive index $n$ of the optical material. A complex $\boldsymbol{P T}$-symmetric potential in quantum mechanics (having a balanced gain and loss of energy) is equivalent to a complex index of refraction (having a balanced gain and loss of optical energy). The early optical experiments used a pair of coupled wave guides in which one wave guide had loss and the other had an equivalent gain [5,6] (see Fig. 3). However, there have now been experiments on multiple wave guides [7], PT-symmetric microwave cavities [8], $\boldsymbol{P} \boldsymbol{T}$-symmetric cavity lasers [9], unidirectional invisibility [10], and optical whispering-gallery resonators [11]. There has also been work on
$\boldsymbol{P T}$-symmetric atomic diffusion [12], superconducting wires [13,14], and $\boldsymbol{P} \boldsymbol{T}$-symmetric electronic circuits [15].

It is remarkable how quickly the initial theoretical and mathematical work on $\boldsymbol{P} \boldsymbol{T}$-symmetric quantum mechanics led to a flurry of beautiful experimental work in diverse areas of physics. (Theoretical and mathematical work in many other areas of physics, such as string theory and supersymmetry, has so far failed to give rise to fruitful experimentation even after decades of research.) It is becoming clear that $\boldsymbol{P} \boldsymbol{T}$ symmetry will have important and lasting practical and commercial applications.

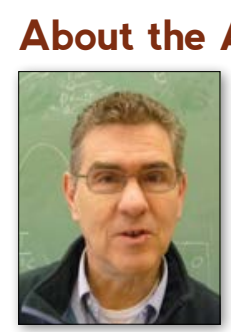

\section{Author}

Carl M. Bender is the Konneker Distinguished Professor of Physics at Washington University and is also visiting Professor at King's College London and Heidelberg University. He received his $\mathrm{AB}$ from Cornell and his $\mathrm{PhD}$ from Harvard. He was awarded Fellowships from the Sloan, Fulbright, Lady Davis, Guggenheim, and Leverhulme Foundations. He was also an Ulam Fellow at Los Alamos National Laboratory and is a Fellow of the APS.

\section{REFERENCES}

[1] C. M. Bender and S. Boettcher, Physical Review Letters 80 , 5243 (1998).

[2] P. E. Dorey, C. Dunning, and R. Tateo, Journal of Physics A 34, 5679 (2001).

[3] C. M. Bender, D. C. Brody, and H. F. Jones, Physical Review Letters 89, 270401 (2002).

[4] R. El-Ganainy, K. Makris, D. N. Christodoulides, and Z. Musslimani, Optics Letters 32, 2632 (2007).

[5] A. Guo, G. J. Salamo, D. Duchesne, R. Morandotti, M. Volatier-Ravat, V. Aimez, G. A. Siviloglou, and D. N. Christodoulides, Physical Review Letters 103, 093902 (2009).

[6] C. E. Rüter, K. G. Makris, R. El-Ganainy, D. N. Christodoulides, M. Segev, and D. Kip, Nature Physics 6, 192 (2010).

[7] A. Regensburger, M.-A. Miri, C. Bersch, J. Näger, G. Onishchukov, D. N. Christodoulides, and U. Peschel, Physical Review Letters 110, 223902 (2013).

[8] S. Bittner, B. Dietz, U. Günther, H. L. Harney, M. Miski-Oglu, A. Richter, and F. Schäfer, Physical Review Letters 108, 024101 (2012).

[9] Y. D. Chong, L. Ge, A. D. Stone, Physical Review Letters 106, 093902 (2011)

[10] Z. Lin, H. Ramezani, T. Eichelkraut, T. Kottos, H. Cao, and D. N. Christodoulides, Physical Review Letters 106, 213901 (2011).

[11] B. Peng, S. K. Özdemir, F. Lei, F. Monifi, M. Gianfreda, G. L. Long, S. Fan, F. Nori, C. M. Bender, L. Yang, Nature Physics 10, 394 (2014).

[12] K. F. Zhao, M. Schaden, and Z. Wu, Phys. Rev. A 81, 042903 (2010).

[13] J. Rubenstein, P. Sternberg, and Q. Ma, Physical Review Letters 99, 167003 (2007).

[14] N. M. Chtchelkatchev, A. A. Golubov, T. I. Baturina, and V. M. Vinokur, Physical Review Letters 109, 150405 (2012).

[15] J. Schindler, A. Li, M. C. Zheng, F. M. Ellis, and T. Kottos, Physical Review A 84, 040101(R) (2011). 